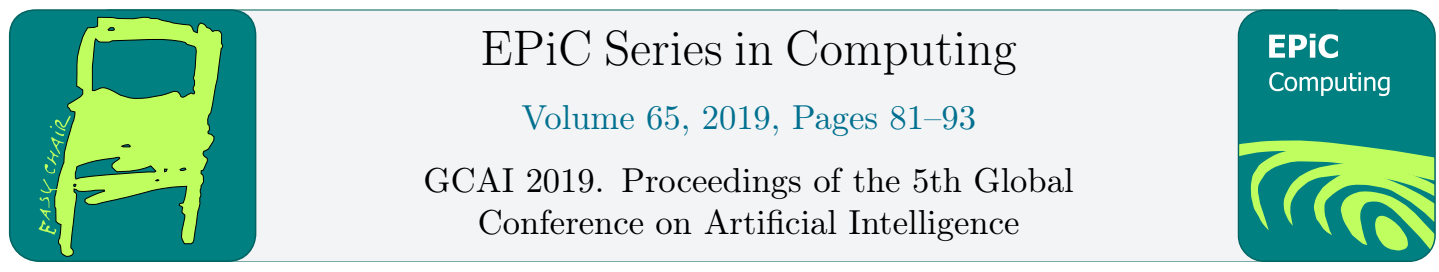

\title{
Projection in a Description Logic of Context with Actions
}

\author{
Satyadharma Tirtarasa and Benjamin Zarrieß \\ Theoretical Computer Science, TU Dresden \\ 01062 Dresden, Germany \\ firstname.lastname@tu-dresden.de
}

\begin{abstract}
Projection is the problem of checking whether the execution of a given sequence of actions will achieve its goal starting from some initial state. In this paper, we study a setting where we combine a two-dimensional Description Logic of context (ConDL) with an action formalism. We choose a well-studied ConDL where both: the possible states of a dynamical system itself (object level) and also different context-dependent views on this system state (context level) are organised in relational structures and can be described using usual DL constructs. To represent how such a system and its views evolve we introduce a suitable action formalism. It allows one to describe change on both levels. Furthermore, the observable changes on the object level due to an action execution can also be contextdependent. We show that the formalism is well-behaved in the sense that projection has the same complexity as standard reasoning tasks in case $\mathcal{A L C O}$ is the underlying DL.
\end{abstract}

\section{Introduction}

The role-based paradigm of modelling languages has been introduced for the design of adaptive and context-sensitive software systems. The concept of roles has been used at different levels of abstraction, for example in data models [6], in a formal high-level modelling language [11] for dynamical systems, and as an extension of more low-level object-oriented programming languages [12]. Unlike in a classical object-oriented setting, where an object has a fixed number of methods attached to it, in a role-based setting an object adapts its behaviour dynamically according to the roles it can play in different contexts. For example, in the conference management system used for this conference the concept of roles is quite prominent. In the context of this conference a researcher might play the role of an author whereas in context of some other conference also the role of a program committee member can be played by the same researcher. Both the view on submissions and the abilities to change something are context-dependent and may vary over time in this scenario.

How to deal with explicit context extensions of modelling languages efficiently is a wellstudied research topic in different areas (e.g. [13, 10, 12, 7]). In [8], Böhme and Lippmann studied a family of contextualized Description Logics (ConDLs). For this family, a reasoning tool has been implemented, and has been used for translating and checking consistency of models of a role-based modelling language for software systems [11, 7].

However, ConDLs are only suitable for expressing static context-dependent knowledge. In this paper, we focus on an extension with dynamic aspects and introduce a ConDL-based action

D. Calvanese and L. Iocchi (eds.), GCAI 2019 (EPiC Series in Computing, vol. 65), pp. 81-93 
formalism for reasoning about change in context models. To talk about particular states we consider the ConDL $\mathcal{A L C O} \llbracket \mathcal{A L C O} \rrbracket$ from [8]. It is a two-sorted logic with a meta level signature for describing contexts and an object level signature for the object domain. $\mathcal{A L C O}$ is the outer meta level logic and is used to describe sets of contexts and relations among them. Each context element of the meta level domain corresponds to a relational structure of the inner object level, which is represented using $\mathcal{A L C O}$ as well. Both levels are connected with a modality that allows one to access the object level from the meta level. In the example of the conference management system one could think of a model, where we talk about researcher accounts with their properties (for example, being PC member or author) and relations (like conflict of interest with someone else) on the meta level, and where each account corresponds to an individual view on concrete submissions and reviews on the object level. The action formalism we introduce makes it possible to describe changes on both levels. For example, the meta level can change if someone becomes a PC member or declares conflict of interest with someone. An object level action might represent the changes when a particular review is entered for a submission. The observable changes of this action from the perspective of a particular account depend on its meta level properties. As a reasoning task we consider the projection problem. Projection is the problem of checking whether the execution of a given sequence of actions will achieve its goal starting from some initial state. In our example, a typical projection query could ask whether, after a subreviewer has accepted to review some submission sub ${ }_{1}$ due to an invitation by a PC member and after a review has been entered by some other PC member for this submission sub ${ }_{1}$, the subreviewer is able to see this review or not. To solve projection we reduce it polynomially to consistency in the underlying logic $\mathcal{A L C O} \llbracket \mathcal{A L C O} \rrbracket$ by adapting techniques that have been used before for reasoning in DL-based action formalisms [5].

The remainder of this paper is structured as follows. In the next section we recall the definitions of $\mathcal{A L C O} \llbracket \mathcal{A L C O} \rrbracket$. Section 3 introduces our action formalism and defines the projection problem. In Section 4, we present our reduction method for deciding projection. We finish with a conclusion in Section 5 .

\section{The Description Logic of Context $\mathcal{A L C O} \llbracket \mathcal{A L C O} \rrbracket$}

For representing context-dependent knowledge we choose $\mathcal{A} \mathcal{L C O} \llbracket \mathcal{A L C O} \rrbracket$, a simple member of the family of ConDLs studied in [8]. To keep this part as simple as possible we focus only on the standard DL $\mathcal{A L C O}$ on both levels. Before defining the two dimensional DL $\mathcal{A L C O} \llbracket \mathcal{A L C O} \rrbracket$ we first briefly recall the basic definitions of standard $\mathcal{A L C O}$. For a thorough introduction to DLs we refer to $[2,3]$.

Definition 1 (Syntax and semantics of $\mathcal{A L C O})$. Let $\mathrm{N}=\left(\mathrm{N}_{\mathrm{C}}, \mathrm{N}_{\mathrm{R}}, \mathrm{N}_{\mathrm{I}}\right)$ be a signature of disjoint sets of concept names, role names and individual names, respectively. Let $A \in \mathrm{N}_{\mathrm{C}}, r \in \mathrm{N}_{\mathrm{R}}$ and $a \in \mathrm{N}_{\mathrm{I}}$. An $\mathcal{A} \mathcal{L C O}$-concept $C$ is built according to the following syntax rule

$$
C::=\top|A|\{a\}|C \sqcap C| \neg C \mid \exists r . C .
$$

Let $C$ and $D$ be $\mathcal{A L C O}$-concept. $A$ general concept inclusion $(G C I)$ is of the form $C \sqsubseteq D$. An $\mathcal{A L C O}-\mathrm{KB} \varphi$ is a Boolean combination of GCIs.

The semantics is defined in terms of an interpretation $\mathcal{I}=\left(\Delta_{\mathcal{I}},{ }^{\mathcal{I}}\right)$ over $\mathrm{N}$, where $\Delta_{\mathcal{I}}$ is the non-empty domain of $\mathcal{I}$ and ${ }^{\mathcal{I}}$ is a mapping that maps each $A \in \mathrm{N}_{\mathrm{C}}$ to a set $A^{\mathcal{I}} \subseteq \Delta_{\mathcal{I}}$, each $r \in \mathrm{N}_{\mathrm{R}}$ to a relation $r^{\mathcal{I}} \subseteq \Delta_{\mathcal{I}} \times \Delta_{\mathcal{I}}$ and each $a \in \mathrm{N}_{1}$ to an element $a^{\mathcal{I}} \in \Delta_{\mathcal{I}}$. We make the unique name assumption, that is, all individual names refer to different domain elements. Furthermore, the mapping ${ }^{\mathcal{I}}$ is extended to complex concepts $C, D$ as follows: $\mathcal{T}^{\mathcal{I}}:=\Delta_{\mathcal{I}},(\{a\})^{\mathcal{I}}:=\left\{a^{\mathcal{I}}\right\}$, 
$(C \sqcap D)^{\mathcal{I}}:=C^{\mathcal{I}} \cap D^{\mathcal{I}},(\neg C)^{\mathcal{I}}:=\Delta_{\mathcal{I}} \backslash C^{\mathcal{I}},(\exists r . C)^{\mathcal{I}}:=\left\{d \in \Delta_{\mathcal{I}} \mid\right.$ there is e $\in \Delta_{\mathcal{I}}$ with $(d, e) \in$ $r^{\mathcal{I}}$ and $\left.e \in C^{\mathcal{I}}\right\}$. The interpretation $\mathcal{I}$ is a model of a $G C I C \sqsubseteq D$ iff $C^{\mathcal{I}} \subseteq D^{\mathcal{I}}$. The definition of a model of a KB $\varphi$ as a Boolean combination of GCIs is defined as usual.

Assume in an example domain about conference management we have a concept name Subs (set of submissions), a role name has-review and an individual sub ${ }_{1}$. We can describe the set of submissions without a review as the $\mathcal{A L C O}$-concept: Subs $\sqcap \neg(\exists$ has-review. $\top)$ and a GCI like $\left\{\right.$ sub $\left._{1}\right\} \sqsubseteq$ Subs $\sqcap \neg(\exists$ has-review. $\top)$ expresses that the individual sub is $_{1}$ an instance of this concept. In the extended logic $\mathcal{A L C O} \llbracket \mathcal{A L C O} \rrbracket$ we are going to define next, one can add an additional level on top which in our example domain could be the level where we talk about PC members, authors, their potential conflicts and their different views on the level of submissions and reviews.

The logic $\mathcal{A L C O} \llbracket \mathcal{A L C O} \rrbracket$ is two-sorted with a meta level signature $\mathrm{M}=\left(\mathrm{M}_{\mathrm{C}}, \mathrm{M}_{\mathrm{R}}, \mathrm{M}_{\mathrm{l}}\right)$ and an object level signature $\mathrm{O}=\left(\mathrm{O}_{\mathrm{C}}, \mathrm{O}_{\mathrm{R}}, \mathrm{O}_{\mathrm{I}}\right)$. We call $\mathrm{M}_{\mathrm{C}}, \mathrm{M}_{\mathrm{R}}$ and $\mathrm{M}_{\mathrm{I}}$ the set of meta concept names, role names, and individual names respectively. Analogously, $\mathrm{O}_{C}, \mathrm{O}_{\mathrm{R}}, \mathrm{O}_{\mathrm{I}}$ is called the set of object concept names, role names, and individual names respectively. All these sets are assumed to be pairwise disjoint. We use m- to denote expressions that use both signatures (e.g. m-concept).

Definition 2 (Syntax of $\mathcal{A L C O} \llbracket \mathcal{A L C O} \rrbracket)$. Let $\varphi$ be an $\mathcal{A L C O}-K B$ over the object level signature $\mathrm{O}$ and $A \in \mathrm{M}_{\mathrm{C}}, r \in \mathrm{M}_{\mathrm{R}}$ and $a \in \mathrm{M}_{\mathrm{I}}$ meta level names. An $\mathcal{A} \mathcal{L C O} \llbracket \mathcal{A L C O} \rrbracket$-meta level concept description $C$ over $\mathrm{M}$ and $\mathrm{O}$ (m-concept for short) is built according to the following syntax rule

$$
C::=A|\{a\}| \llbracket \varphi \rrbracket|C \sqcap C| \neg C \mid \exists r . C .
$$

Further constructors are defined as abbreviations: $\top:=\neg(A \sqcap \neg A)$ and $\perp:=(A \sqcap \neg A)$ (for some $\left.A \in \mathrm{M}_{\mathrm{C}}\right), C \sqcup D:=\neg(C \sqcap \neg D)$ and $\forall r . C:=\neg \exists r . \neg C$.

Let $C$ and $D$ be m-concepts. An $\mathcal{A L C O} \llbracket \mathcal{A L C O} \rrbracket$-Boolean meta level knowledge base $\psi$ over $\mathrm{M}$ and $\mathrm{O}$ ( $m$-KB for short) is built according to the following syntax rule

$$
\psi::=C \sqsubseteq D|\psi \wedge \psi| \neg \psi .
$$

Notation for concept assertions and role assertions is used as abbreviations: $(a: C):=\{a\} \sqsubseteq C$ and $((a, b): r):=\{a\} \sqsubseteq \exists r .\{b\}$. Further Boolean connectives like $\vee$ and $\rightarrow$ are defined as usual.

The only non-standard expression is the meta concept constructor $\llbracket \varphi \rrbracket$ that refers to a standard $\mathcal{A L C O}-\mathrm{KB} \varphi$. It gives access to the object level from the meta level and describes the set of meta level domain elements (contexts) in which $\varphi$ is true.

The semantics of $\mathcal{A L C O} \llbracket \mathcal{A L C O} \rrbracket$ is defined in terms of nested interpretations. The structure consists of a single meta level interpretation over $\mathrm{M}$ where each domain element is associated with an object level interpretation over $\mathrm{O}$ over a rigid domain $\Delta$.

Definition 3 (Nested Interpretation). A nested interpretation $\mathfrak{I}$ (over $\mathrm{M}$ and $\mathrm{O}$ ) is a tuple of the form $\mathfrak{I}:=\left(\mathbb{C},{ }^{\mathfrak{I}}, \Delta,\left\{\mathcal{I}_{c}\right\}_{c \in \mathbb{C}}\right)$, where

- $\left(\mathbb{C}, \cdot^{\mathfrak{I}}\right)$ is an $\mathrm{M}$-interpretation, and

- $\mathcal{I}_{c}:=\left(\Delta,{ }^{\mathcal{I}_{c}}\right)$ is an O-interpretation for each $c \in \mathbb{C}$.

Definition 4 (Semantics). Let $\mathfrak{I}=\left(\mathbb{C}, \cdot^{\mathfrak{I}}, \Delta,\left\{\mathcal{I}_{c}\right\}_{c \in \mathbb{C}}\right)$ be a nested interpretation. The extension of the mapping.${ }^{\mathfrak{I}}$ to complex $\mathrm{m}$-concepts is defined by induction on the structure of $m$-concepts 
$C$ and $D$ as follows:

$$
\begin{aligned}
& (\{a\})^{\mathfrak{I}} \quad:=\left\{a^{\mathfrak{I}}\right\} ; \\
& (\llbracket \varphi \rrbracket)^{\mathfrak{I}} \quad:=\left\{c \in \mathbb{C} \mid \mathcal{I}_{c} \models \varphi\right\} ; \\
& (C \sqcap D)^{\mathfrak{I}}:=C^{\mathfrak{I}} \cap D^{\mathfrak{I}} \\
& (\neg C)^{\mathfrak{I}} \quad:=\mathbb{C} \backslash C^{\mathfrak{I}} ; \\
& (\exists r . C)^{\mathfrak{I}} \quad:=\left\{c \in \mathbb{C} \mid \text { there exists } c^{\prime} \in \mathbb{C} \text { such that }\left(c, c^{\prime}\right) \in r^{\mathfrak{I}} \text { and } c^{\prime} \in C^{\mathfrak{I}}\right\}
\end{aligned}
$$

where $a \in \mathrm{M}_{\mathrm{l}}, r \in \mathrm{M}_{\mathrm{R}}$ and $\varphi$ is an $\mathcal{A L C O}-K B$ over $\mathrm{O}$.

Let $\psi$ be an $m-K B$. Satisfaction of $\psi$ in $\mathfrak{I}$, written as $\mathfrak{I}=\psi$ ( $\mathfrak{I}$ is a model of $\psi$ ), is defined by induction on the structure of $\psi$ as follows:

$$
\begin{array}{ll}
\mathfrak{I} \models C \sqsubseteq D & \text { iff } C^{\mathfrak{I}} \subseteq D^{\mathfrak{I}} ; \\
\mathfrak{I} \models \psi_{1} \wedge \psi_{2} & \text { iff } \mathfrak{I}=\psi_{1} \text { and } \mathfrak{I}=\psi_{2} ; \\
\mathfrak{I} \models \neg \psi_{1} \quad \text { iff } \mathfrak{I} \not \psi_{1} .
\end{array}
$$

Example 1. We describe some aspects of a conference management domain. On the meta level we talk about accounts that can be PC members of a conference (meta level concept name PC) with possibly conflict of interest (meta level role has-conflict) to authors (concept name Author). Each account has a particular view on the object level where we have a domain of submissions and reviews. The object level concept names Subs-To-Review and Own-Subs describe the assigned submissions for reviewing and their own written submissions, respectively. The object level role has-review relates submissions to their reviews. We describe an initial situation using the meta level individual names bob's-account and alice's-account and the object level name sub 1 denoting a concrete submission. Intuitively, in this model the meta level concept

$$
\llbracket \mathrm{sub}_{1}: \text { Own-Subs》 }
$$

describes the set of accounts (meta level domain elements) in which sub sis $_{1}$ an instance of

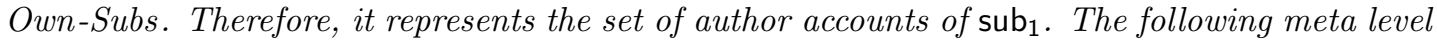
axioms represent some initial knowledge:

$$
\begin{aligned}
& \text { alice 's-account : }\left(\forall \text { has-conflict. } \neg \llbracket \text { sub }_{1}: \text { Own-Subs } \rrbracket\right) \\
& \text { bob's-account }: \text { : } \text { sub }_{1}: \text { Own-Subs } \rrbracket \\
& T \sqsubseteq \llbracket \text { sub }_{1}: \forall \text { has-review. } \perp \rrbracket \\
& \text { Author } \equiv \neg \llbracket \text { Own-Subs } \sqsubseteq \perp \rrbracket \\
& \neg \llbracket \text { Subs-To-Review } \sqsubseteq \perp \rrbracket \sqsubseteq \mathrm{PC}
\end{aligned}
$$

Alice has no conflict of interest with an author of the submission sub $_{1}$ (1). Bob is an author of sub $_{1}(2)$, which has not received any reviews yet (3). Author accounts are defined as those accounts with own submissions (4). Only PC members are allowed to review (5).

We show a model (nested interpretation) of axioms (1) - (5) in Figure 1. It shows the meta level with $P C$ members and authors on the left labelled with $\mathbb{C}$. For the sake of conciseness, we use alice and bob to denote alice's-account and bob's-account. These are the two named accounts in our domain. In this particular model alice is a PC member with a conflict of interest to some unnamed account. In the middle and on the right of the figure the particular views of bob and alice on submissions and reviews are shown. 


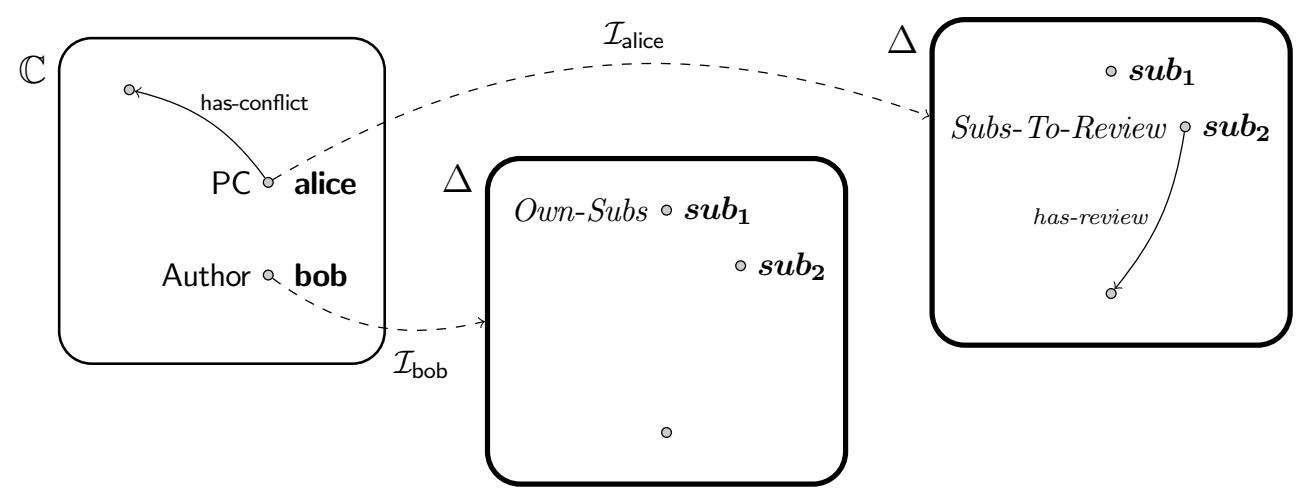

Figure 1: A model of axioms 1-5

\section{Representing Context-dependent Change}

We define separate action descriptions for the object level and the meta level. Syntactically, action descriptions are complex expressions with constructors for describing conditional and simultaneous execution. Semantically, actions update interpretations by changing the membership of named individuals in concept names or of pairs of named individuals in role names depending on the satisfaction of the condition.

Definition 5. Let $\psi$ be an $m-K B$ and $A \in \mathrm{M}_{\mathrm{C}}, r \in \mathrm{M}_{\mathrm{R}}$ and $a, b \in \mathrm{M}_{\mathrm{I}}$ be meta level names. An $\mathrm{M}$-action description $\alpha$ (M-action for short) is built according to the following syntax rule:

$$
\alpha:=\langle A \oplus a\rangle|\langle A \ominus a\rangle|\langle r \oplus(a, b)\rangle|\langle r \ominus(a, b)\rangle|(\psi \triangleright \alpha) \mid(\alpha \| \alpha) .
$$

Let $C$ be an m-concept and $B \in \mathrm{O}_{\mathrm{C}}, s \in \mathrm{O}_{\mathrm{R}}$ and $o, o^{\prime} \in \mathrm{O}_{\mathrm{I}}$ object level names. An $\mathrm{O}$-action description $\beta$ (O-action for short) is built according to the following syntax rule:

$$
\beta:=\langle B \oplus o\rangle|\langle B \ominus o\rangle|\left\langle s \oplus\left(o, o^{\prime}\right)\right\rangle\left|\left\langle s \ominus\left(o, o^{\prime}\right)\right\rangle\right|(C \triangleright \beta) \mid(\beta \| \beta) .
$$

We write just action if we do not distinguish between $\mathrm{M}$-actions and $\mathrm{O}$-actions. Actions of the form $\langle A \oplus a\rangle,\langle r \oplus(a, b)\rangle,\langle B \oplus o\rangle$ or $\left\langle s \oplus\left(o, o^{\prime}\right)\right\rangle$ are called atomic actions.

An atomic action like $\langle A \oplus a\rangle$ over some signature $\mathrm{N}$ changes an $\mathrm{N}$-interpretation $\mathcal{I}$ by adding $a^{\mathcal{I}}$ to $A^{\mathcal{I}}$ and $\langle r \ominus(a, b)\rangle$ deletes $\left(a^{\mathcal{I}}, b^{\mathcal{I}}\right)$ from $r^{\mathcal{I}}$. M-actions have m-KBs as conditions and $\mathrm{O}$-actions have $\mathrm{m}$-concepts as conditions. A conditional $\mathrm{M}$-action $\left(\psi \triangleright \alpha_{1}\right)$ takes effect in the meta-level interpretation only if $\psi$ is satisfied and a conditional O-action of the form $C \triangleright \beta_{1}$ means that an O-interpretation $\mathcal{I}_{c}$ in a nested interpretation $\mathfrak{I}$ is only updated with $\beta_{1}$ if $c$ belongs to $C$ in $\mathfrak{I}$. The construct $\left(\alpha_{1} \| \alpha_{2}\right)$ means that $\alpha_{1}$ and $\alpha_{2}$ are executed simultaneously, similar with a conjunction.

For the first step of the definition of the execution semantics we define how a set of atomic $\mathrm{M}$-actions (or atomic O-actions) updates a non-nested $\mathrm{M}$-interpretation (or O-interpretation). Both levels behave similarly if atomic actions are considered.

Definition 6 (Update). Let $\mathrm{N} \in\{\mathrm{M}, \mathrm{O}\}$ denote either the meta-level or object-level signature, and let $\mathcal{I}:=\left(\Delta_{\mathcal{I}},{ }^{\mathcal{I}}\right)$ be an $\mathrm{N}$-interpretation and $\mathfrak{E}$ a set of atomic $\mathrm{N}$-actions. The update of $\mathcal{I}$ with $\mathfrak{E}$ is an interpretation denoted by $\mathcal{I}^{\mathfrak{E}}$ and is defined for all $A \in \mathrm{N}_{\mathrm{C}}$, all $r \in \mathrm{N}_{\mathrm{R}}$ and all 
$a \in \mathrm{N}_{\mathrm{I}}$ as follows

$$
\begin{aligned}
& \Delta_{\mathcal{I}^{\mathfrak{E}}}:=\Delta_{\mathcal{I}} \\
& A^{\mathcal{I}^{\mathfrak{E}}}:=\left(A^{\mathcal{I}} \backslash\left\{a^{\mathcal{I}} \mid\langle A \ominus a\rangle \in \mathfrak{E}\right\}\right) \cup\left\{b^{\mathcal{I}} \mid\langle A \oplus b\rangle \in \mathfrak{E}\right\} \\
& r^{\mathcal{I}^{\mathfrak{E}}}:=\left(r^{\mathcal{I}} \backslash\left\{\left(a^{\mathcal{I}}, b^{\mathcal{I}}\right) \mid\langle r \ominus(a, b)\rangle \in \mathfrak{E}\right\}\right) \cup\left\{\left(a^{\mathcal{I}}, b^{\mathcal{I}}\right) \mid\langle r \oplus(a, b)\rangle \in \mathfrak{E}\right\} \\
& a^{\mathcal{I}^{\mathfrak{E}}}:=a^{\mathcal{I}} .
\end{aligned}
$$

Next, we define the effects of the execution of a complex action in a nested interpretation as a set of atomic actions. The definition tells us how the conditions attached by the " $\triangleright$ " operator are evaluated for $\mathrm{M}$-actions and $\mathrm{O}$-actions.

Definition 7 (Effects). Let $\mathfrak{I}=\left(\mathbb{C},{ }^{\mathfrak{I}}, \Delta,\left\{\mathcal{I}_{c}\right\}_{c \in \mathbb{C}}\right)$ be a nested interpretation and $\alpha$ an $\mathrm{M}$ action. The set of atomic $\mathrm{M}$-actions for $\mathfrak{I}$ and $\alpha$, denoted by $\mathcal{E}(\alpha, \mathfrak{I})$, is defined by induction on the structure of $\alpha$ as follows

$$
\begin{aligned}
& \mathcal{E}(\langle A \oplus a\rangle, \mathfrak{I}):=\{\langle A \oplus a\rangle\} \quad \text { and } \quad \mathcal{E}(\langle r \oplus(a, b)\rangle, \mathfrak{I}):=\{\langle r \oplus(a, b)\rangle\} \\
& \mathcal{E}\left(\psi \triangleright \alpha_{1}, \mathfrak{I}\right):= \begin{cases}\mathcal{E}\left(\alpha_{1}, \mathfrak{I}\right) & \text { if } \mathfrak{I} \models \psi, \\
\emptyset & \text { otherwise }\end{cases} \\
& \mathcal{E}\left(\alpha_{1} \| \alpha_{2}, \mathfrak{I}\right):=\mathcal{E}\left(\alpha_{1}, \mathfrak{I}\right) \cup \mathcal{E}\left(\alpha_{2}, \mathfrak{I}\right) .
\end{aligned}
$$

Let $\beta$ be an $\mathrm{O}$-action and $c \in \mathbb{C}$. The set of sets of atomic $\mathrm{O}$-actions for $\mathfrak{I}, c$ and $\beta$, denoted by $\mathcal{E}(\beta, c, \mathfrak{I})$, is defined by induction on the structure of $\beta$ as follows

$$
\begin{aligned}
& \mathcal{E}(\langle B \oplus o\rangle, c, \mathfrak{I}):=\{\langle B \oplus o\rangle\} \quad \text { and } \quad \mathcal{E}\left(\left\langle s \oplus\left(o, o^{\prime}\right)\right\rangle, c, \mathfrak{I}\right):=\left\{\left\langle s \oplus\left(o, o^{\prime}\right)\right\rangle\right\} \\
& \mathcal{E}\left(C \triangleright \beta_{1}, c, \mathfrak{I}\right):= \begin{cases}\mathcal{E}\left(\beta_{1}, c, \mathfrak{I}\right) & \text { if } c \in C^{\mathfrak{I}}, \\
\emptyset & \text { otherwise; }\end{cases} \\
& \mathcal{E}\left(\beta_{1} \| \beta_{2}, c, \mathfrak{I}\right):=\mathcal{E}\left(\beta_{1}, c, \mathfrak{I}\right) \cup \mathcal{E}\left(\beta_{2}, c, \mathfrak{I}\right) .
\end{aligned}
$$

An M-action only updates the outer meta level interpretation of a nested interpretation. An O-action leaves the meta level interpretation unchanged and updates all object level interpretation simultaneously. How a particular object level interpretation $\mathcal{I}_{c}$ in a nested interpretation $\mathfrak{I}$ is updated as the result of executing an O-action $\beta$ depends only on the membership of $c$ in the meta level concepts used as conditions in $\beta$. The corresponding set $\mathcal{E}(\beta, c, \mathfrak{I})$ can also be empty.

Definition 8 (Nested Update). Let $\mathfrak{I}:=\left(\mathbb{C},{ }^{\mathfrak{I}}, \Delta,\left\{\mathcal{I}_{c}\right\}_{c \in \mathbb{C}}\right)$ be some nested interpretation, $\alpha$ an $\mathrm{M}$-action and $\beta$ an $\mathrm{O}$-action. The update of $\mathfrak{I}$ with $\alpha$ is the nested interpretation

$$
\mathfrak{I}^{\alpha}:=\left(\mathbb{C}, \cdot^{\Im^{\alpha}}, \Delta,\left\{\mathcal{I}_{c}\right\}_{c \in \mathbb{C}}\right),
$$

where $\left(\mathbb{C}, \Im^{\alpha}\right)$ is the update of $\left(\mathbb{C}, \cdot^{\mathfrak{I}}\right)$ with $\mathcal{E}(\alpha, \mathfrak{I})$ and all other components are unchanged. The update of $\mathfrak{I}$ with $\beta$ is the nested interpretation

$$
\mathfrak{I}^{\beta}:=\left(\mathbb{C}, \cdot^{\mathfrak{I}}, \Delta,\left\{\mathcal{J}_{c}\right\}_{c \in \mathbb{C}}\right),
$$

where for each $c \in \mathbb{C}$ the $\mathrm{O}$-interpretation $\mathcal{J}_{c}:=\left(\Delta, \mathcal{J}_{c}\right)$ is the update of $\mathcal{I}_{c}$ with $\mathcal{E}(\beta, c, \mathfrak{I})$.

Let $\sigma$ be a sequence of $\mathrm{M}$-actions and $\mathrm{O}$-actions the update $\mathfrak{I}^{\sigma}$ is defined in the obvious way 

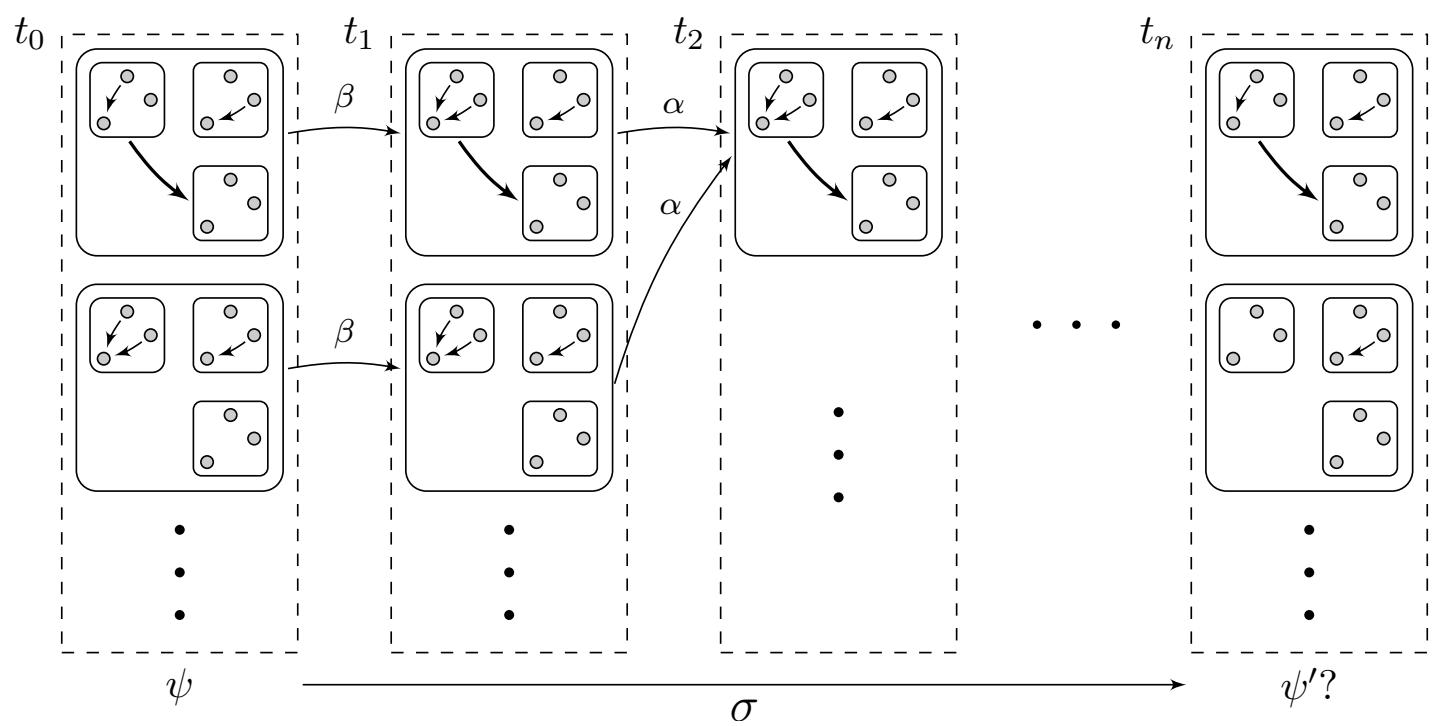

Figure 2: A depiction of the projection problem

by induction on $\sigma$.

As in [1] the semantics of actions is defined independent from global constraints formulated as GCIs. Checking whether or not a given sequence of actions preserves certain GCIs is viewed as a reasoning task (called projection problem). Preserving global GCIs is not something we try to enforce in the semantics. For a discussion on this view on state constraints we refer to [9].

Note that it is possible to write an action that adds and deletes an object (or a pair of objects) to and from a name simultaneously. The semantics of updates gives precedence to add effects but we want to exclude those descriptions. In the following we assume that for any $\mathrm{M}$-action $\alpha$, any $\mathrm{O}$-action $\beta$ and any nested interpretation $\mathfrak{I}$ and meta level domain element $c$ the sets $\mathcal{E}(\alpha, \mathfrak{I})$ and $\mathcal{E}(\beta, c, \mathfrak{I})$ are non-contradictory, i.e., does not contain both $\langle A \oplus a\rangle$ and $\langle A \ominus a\rangle$ and analogously for roles.

We are interested in checking whether a certain consequence formulated as an m-KB holds after executing a sequence of actions given an incomplete representation of the initial state in terms of an $\mathrm{m}-\mathrm{KB}$.

Definition 9 (Projection). Let $\psi, \psi^{\prime}$ be $m$-KBs and $\sigma$ a sequence of actions. We say that $\psi^{\prime}$ is a consequence of executing $\sigma$ in $\psi$ iff for all models $\mathfrak{I}$ of $\psi$, we have that $\mathfrak{I}^{\sigma} \models \psi^{\prime}$. The projection problem is then to decide whether $\psi^{\prime}$ is a consequence of executing $\sigma$ in $\psi$.

We depict a sketch of the projection problem in Figure 2. All possible initial models that satisfy $\psi$ are contained in $t_{0}$. Then, an $\mathrm{O}$-action $\beta$ is executed and changes each nestedinterpretation into the new one in $t_{1}$ with corresponding effects of $\beta$. Then, an M-action is executed and changes them in a similar way to $t_{2}$. It is possible that two nested-interpretations collapse into one due to the effect (e.g. from $t_{1}$ to $t_{2}$ ). Furthermore, our formalization is deterministic, i.e., there is only one successor for each nested-interpretation. After executing all actions in sequence $\sigma$, we have the final sets of possible nested-interpretations in $t_{n}$. Projection is the problem to check whether all of them satisfy properties that are represented by an m-KB $\psi^{\prime}$. 
We continue our example about the conference management system.

Example 2 (Example 1 continued). An M-action for adding Alice as a PC Member of the conference is given by

$$
\text { add-pc : }=\langle\mathrm{PC} \oplus \text { alice's-account }\rangle .
$$

It updates a nested interpretation by adding alice's-account to PC on the meta level and changing nothing else. Next, we want to define an action which assigns Alice as a reviewer for the submission sub $_{1}$ under the condition that she has no conflict of interest with an author of this submission. It is specified as an O-action:

$$
\begin{aligned}
\text { add-sub : }= & \left(\{\text { alice's-account }\} \sqcap \neg \exists \text { has-conflict. } \llbracket \text { sub }_{1}: \text { Own-Subs } \rrbracket\right) \triangleright \\
& \left\langle\text { Subs-To-Review } \oplus \text { sub }_{1}\right\rangle
\end{aligned}
$$

Note that only the account of Alice is affected. The action only updates the object level interpretation associated with alice's-account by adding sub s $_{1}$ to the review set (Subs-To-Review).

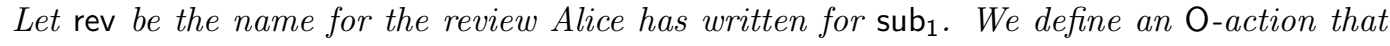
enters this review to the system and removes sub $_{1}$ from the review list of Alice simultaneously.

$$
\begin{aligned}
\text { finish } & :=\text { enter } \| \text { remove; } \\
\text { enter } & :=\left(\mathrm{PC} \sqcap \neg \exists \text { has-conflict. } \llbracket \text { sub }_{1}: \text { Own-Subs } \rrbracket\right) \triangleright\left\langle\text { has-review } \oplus\left(\text { sub }_{1}, \text { rev }\right)\right\rangle ; \\
\text { remove } & :=\{\text { alice's-account }\} \triangleright\langle\text { Subs-To-Review } \ominus s\rangle .
\end{aligned}
$$

The review rev is only visible for PC members with no conflict of interest with someone that is an author of sub.

Assume initially we have axioms (1)-(5) from Example 1. After performing the sequence add-pc; add-sub it holds that

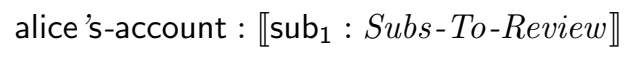

is true and the constraint

$$
\neg \llbracket \text { Subs-To-Review } \sqsubseteq \perp \rrbracket \sqsubseteq \mathrm{PC}
$$

is preserved. Furthermore, after add-pc; add-sub; finish we have that

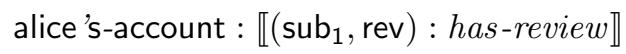

is true.

\section{Deciding the Projection Problem in $\mathcal{A L C O} \llbracket \mathcal{A L C O} \rrbracket$}

The approach of solving the projection problem in a DL-based action formalism by reducing it to a standard consistency problem in the underlying DL has been applied already in several settings (e.g. $[5,4,14])$. The overall idea we use here is similar to previous techniques extended to nested structures in our case.

As a first step we introduce a normal form of action descriptions by conjoining conditions and pushing them inside. We say that an $\mathrm{N}$-action $\mu$ with $\mathrm{N} \in\{\mathrm{M}, \mathrm{O}\}$ is in normal form if it 
is of the form

$$
\left(\psi_{1} \triangleright e_{1}\right)\|\ldots\|\left(\psi_{n} \triangleright e_{n}\right),
$$

where each $e_{i}$, for any $i, 1 \leq i \leq n$ is an atomic $\mathrm{N}$-action and $\psi_{i}$ is either an m-KB (in case of an $\mathrm{M}$-action) or an $\mathrm{m}$-concept (in case of an $\mathrm{O}$-action). We normalize an arbitrary $\mathrm{N}$-action by applying exhaustively the following rules:

$$
\psi_{1} \triangleright\left(\psi_{2} \triangleright \mu\right) \rightsquigarrow\left(\psi_{1} \star \psi_{2}\right) \triangleright \mu \quad \psi \triangleright\left(\mu_{1} \| \mu_{2}\right) \rightsquigarrow\left(\psi \triangleright \mu_{1}\right) \|\left(\psi \triangleright \mu_{2}\right),
$$

where $\star \in\{\wedge, \sqcap\}$ stands for $\wedge$ in case of an M-action and for $\sqcap$ in case of an O-action. W.l.o.g., we assume from now on that any action is in normal form. For convenience, we denote a normal form of an $\mathrm{N}$-action $\mu$ as a set of atomic $\mathrm{N}$-actions with a single condition attached: $\mu=\left\{\left(\psi_{1} \triangleright e_{1}\right), \ldots,\left(\psi_{n} \triangleright e_{n}\right)\right\}$. Although the right side seems causing an exponential blow-up, notice that the number of copies of the conditions $(\psi$ or $C)$ are polynomially bounded by the number of atomic effects.

Let an $\mathrm{m}-\mathrm{KB} \psi$ (initial state), $\psi^{\prime}$ (goal state) and a sequence of actions $\sigma=\mu_{1}, \ldots, \mu_{n}$ in normal form be the input of the projection problem. Our goal is to construct a reduction $m-K B$ that is consistent iff $\psi^{\prime}$ is a consequence of executing $\sigma$ in $\psi$.

We say that concepts, roles, and individuals are relevant if they occur in the input of the projection problem. For the reduction we use fresh concept names and role names of the corresponding sort. For each execution step $0 \leq i \leq n$, we introduce fresh time-stamped copies $A^{(i)}$ of all relevant concept names, $r^{(i)}$ of all relevant role names, and fresh time-stamped concept names $T_{C}^{(i)}$ for every relevant complex subconcept $C . A^{(0)}$ refers to the initial content of $A$ and the further copies $A^{(j)}, 1 \leq j \leq n$ refer only to the set of named individual names of the corresponding sort that are instance of $A$ after the $j$ th execution step. This holds for both concept and role names. The copies of the form $T_{C}^{(i)}$ represent the content (both named and unnamed) of the complex concept $C$ after the $i$ th execution step. The distinction between named and unnamed is made because actions only affect named individuals.

Furthermore, for the set of all named individuals of sort object in the input (denoted by $\left.\mathrm{Obj}_{\mathrm{O}}\right)$ and for the set of all named meta level individuals in the input $\left(\mathrm{Obj}_{\mathrm{M}}\right)$ two fresh concept names $N_{\mathrm{O}}$ and $N_{\mathrm{M}}$, respectively, are introduced.

The meaning of the new names is now axiomatized using meta level axioms as follows. For $N_{\mathrm{O}}$ and $N_{\mathrm{M}}$ we have

$$
\psi_{\mathrm{obj}}=\left(N_{\mathrm{M}} \equiv \bigsqcup_{c \in \mathrm{Obj}_{\mathrm{M}}}\{c\}\right) \wedge\left(\top \sqsubseteq \llbracket N_{\mathrm{O}} \equiv \bigsqcup_{a \in \mathrm{Obj}_{\mathrm{o}}}\{a\} \rrbracket\right) .
$$

We use $\tau(C, i)$ to denote the concept definition we introduce to define the names of the form $T_{C}^{(i)}$. It is defined by induction on the structure of $C$ as follows:

$$
\begin{gathered}
T_{A}^{(i)} \equiv\left(N_{\mathrm{O}} \sqcap A^{(i)}\right) \sqcup\left(\neg N_{\mathrm{O}} \sqcap A^{(0)}\right) \\
T_{\{a\}}^{(i)} \equiv\{a\} \quad T_{\neg C}^{(i)} \equiv \neg T_{C}^{(i)} \quad T_{C_{1} \sqcap C_{2}}^{(i)} \equiv T_{C_{1}}^{(i)} \sqcap T_{C_{2}}^{(i)} \\
T_{\exists r . C}^{(i)} \equiv\left(N_{\mathrm{O}} \sqcap\left(\left(\exists r^{(0)} .\left(\neg N_{\mathrm{O}} \sqcap T_{C}^{(i)}\right)\right) \sqcup\left(\exists r^{(i)} .\left(N_{\mathrm{O}} \sqcap T_{C}^{(i)}\right)\right)\right) \sqcup \sqcup\left(\neg N_{\mathrm{O}} \sqcap \exists r^{(0)} . T_{C}^{(i)}\right)\right.
\end{gathered}
$$

For referring meta concepts we have $T_{\llbracket \varphi \rrbracket}^{(i)} \equiv \llbracket \varphi^{(i)} \rrbracket$. For the object level, we ensure that the 
concept definitions hold in any context:

$$
\psi_{\text {defo }_{\mathrm{O}}}^{(i)}:=\bigwedge_{C \in \mathcal{R}_{\mathrm{O}}} \top \sqsubseteq \llbracket \tau(C, i) \rrbracket \quad \psi_{\mathrm{def}_{\mathrm{M}}}^{(i)}:=\bigwedge_{G \in \mathcal{R}_{\mathrm{M}}} \tau(G, i),
$$

where $\mathcal{R}_{\mathrm{O}}$ is the set of relevant object level subconcepts and $\mathcal{R}_{\mathrm{M}}$ the analogous set on the meta level. Given an O-GCI $\gamma=C \sqsubseteq D$ and a timestamp $0 \leq i \leq n$, we define the timestamped copy $\gamma^{(i)}:=T_{C}^{(i)} \sqsubseteq T_{D}^{(i)}$, and $\varphi^{(i)}$ is the timestamped O-KB obtained from $\varphi$ as the result of replacing every O-GCI $\gamma$ in $\varphi$ with $\gamma^{(i)}$. Timestamped copies for M-GCI $\zeta^{(i)}$ and m-KB $\psi^{(i)}$ are defined analogously.

We simply put timestamp zero for the initial knowledge base $\psi$, i.e., we include $\psi^{(0)}$ as a conjunct of the reduction $\mathrm{m}-\mathrm{KB}$.

Next, we encode the effects of each action $\mu_{i}$ using the m-KB $\psi_{\text {act }_{i}}$. Intuitively, we make sure that if the condition is satisfied, then corresponding atomic actions are applied to the next step. We define corresponding assertions for each atomic action $e$ and a timestamp $i$ with $\pi(e, i)$.

$$
\begin{aligned}
\pi(\langle A \oplus a\rangle, i):=\left(a: A^{(i)}\right) & \pi(\langle A \ominus a\rangle, i):=\left(a: \neg A^{(i)}\right) \\
\pi(\langle r \oplus(a, b)\rangle, i):=\left((a, b): r^{(i)}\right) & \pi(\langle r \ominus(a, b)\rangle, i):=\left((a, b): \neg r^{(i)}\right)
\end{aligned}
$$

We distinguish two cases, whether the action is an $\mathrm{M}$-action or an O-action. First, we consider the case of $\mu_{i}$ is an $\mathrm{M}$-action $\alpha_{i}$. We define:

$$
\psi_{\mathrm{act}_{\mathrm{M}}}^{(i)}:=\bigwedge_{\psi \triangleright e \in \alpha_{i}}\left(\psi^{(i-1)} \rightarrow \pi(e, i)\right)
$$

We encode the atomic O-actions similarly for $\mu_{i}=\beta_{i}$, with taking the context into account. Instead of having an $\mathrm{m}-\mathrm{KB}$, we have a timestamped $\mathrm{m}$-concept as the condition. The atomic $\mathrm{O}$-actions are propagated using referring meta concept for those contexts.

$$
\psi_{\text {acto }}^{(i)}:=\bigwedge_{G \triangleright e \in \beta_{i}}\left(T_{G}^{(i-1)} \sqsubseteq \llbracket \pi(e, i) \rrbracket\right)
$$

In case of the other type of action happens at timestamp $i$, the corresponding $\psi_{\text {act }}^{(i)}$ is simply $\top$. For example, $\psi_{\mathrm{act}_{\mathrm{M}}}^{(i)}=\top$ if $\mu_{i}$ is an O-action.

Then, we make sure a change only happens if there is an effect that enforces it. For every $i, 1 \leq i \leq n$, we define an $\mathrm{m}-\mathrm{KB} \psi_{\text {min }_{\mathrm{M}}}^{(i)}$ that encodes all the non-effects, that is, for each named individual $a$, each relevant concept name $A$ from the meta level it contains a conjunct of the form

$$
\begin{aligned}
& \left(\left(a: A^{(i-1)} \wedge \bigwedge_{\psi \triangleright\langle A \ominus a\rangle \in \mu_{i}} \neg \psi^{(i-1)}\right) \rightarrow a: A^{(i)}\right) \wedge \\
& \left(a: \neg A^{(i-1)} \wedge \bigwedge_{\psi \triangleright\langle A \oplus a\rangle \in \mu_{i}} \neg \psi^{(i-1)}\right) \rightarrow a: \neg A^{(i)},
\end{aligned}
$$

and analogous conjuncts for all relevant role names and pairs of named individuals. Likewise, we ensure a minimization of changes on the object level with an m-KB $\psi_{\min }^{(i)}$ for every $i, 1 \leq i \leq n$ 
consisting of conjuncts of the form

$$
\left(\llbracket o: B^{(i-1)} \rrbracket \wedge \bigwedge_{G \triangleright\langle B \ominus o\rangle \in \mu_{i}} \neg T_{G}^{(i-1)}\right) \rightarrow \llbracket o: B^{(i)} \rrbracket,
$$

and analogously for the negative case and role names. If no condition in the effects that might change an assertion are satisfied, then they should stay the same. Finally, we define the complete reduction $\mathrm{m}-\mathrm{KB}$ :

$$
\begin{array}{rlll}
\psi_{\text {red }}:= & \wedge \psi_{\text {init }} & \wedge \bigwedge_{0 \leq i \leq n} \psi_{\text {defo }_{0}}^{(i)} & \wedge \bigwedge_{0 \leq i \leq n} \psi_{\text {def }_{\mathrm{M}}}^{(i)} \wedge \\
\bigwedge_{1 \leq i \leq n} \psi_{\text {acto }}^{(i)} & \wedge \bigwedge_{1 \leq i \leq n} \psi_{\text {act }}^{(i)} & \wedge \bigwedge_{1 \leq i \leq n} \psi_{\text {mino }}^{(i)} & \wedge \bigwedge_{1 \leq i \leq n} \psi_{\min }^{(i)}
\end{array}
$$

Lemma 1. Let $\psi$ be an $m-K B, \sigma=\mu_{1}, \ldots, \mu_{n}$ be a sequence of actions, and $\psi_{\text {red }}$ be defined as above. The following properties hold:

1. For every sequence of nested-interpretations $\mathfrak{I}_{0}, \ldots, \mathfrak{I}_{n}$ such that $\mathfrak{I}_{0} \models \psi$ and $\mathfrak{I}_{i}=\mathfrak{I}_{i-1}^{\mu_{i}}$ for each $i, 1 \leq i \leq n$ there exists an interpretation $\mathfrak{L}=\psi_{\text {red }}$ such that for every $i, 0 \leq i \leq n$, and every relevant $m-K B \psi$, we have $\mathfrak{I}_{i} \models \psi$ iff $\mathfrak{L} \models \psi^{(i)}$.

2. For every nested-interpretation $\mathfrak{L}=\psi_{\text {red }}$, there exists a sequence of nested-interpretations $\mathfrak{I}_{0}, \ldots, \mathfrak{I}_{n}$ such that $\mathfrak{I}_{0}=\psi$ and $\mathfrak{I}_{i}=\mathfrak{I}_{i-1}^{\mu_{i}}$ for every $i, 1 \leq i \leq n$ such that for every $i$, $0 \leq i \leq n$, and every relevant $m-K B \psi$, we have $\mathfrak{I}_{i}=\psi$ iff $\mathfrak{L}=\psi^{(i)}$.

Proof. (Sketch)

1. We build a model $\mathfrak{L}$ by interpreting $i$-timestamped copies of concepts (roles) in the same way with the concepts (roles) in $\mathfrak{I}^{(i)}$. Obviously, we need to employ the idea on both meta and object levels. Then, what remains is to show that $\mathfrak{L}$ is indeed a model of $\psi_{\text {red }}$. The $\psi_{\text {init }}$ and $\psi_{\text {obj }}$ parts are trivial. Next, we need an intermediate claim that shows that we irrelevant individuals will not change w.r.t. concept and role membership due to the formalization. Using such claim, we show that each concept definition of $T_{C}^{(i)}$ in $\bigwedge_{0 \leq i \leq n} \psi_{\text {defo }_{0}}^{(i)} \wedge \bigwedge_{0 \leq i \leq n} \psi_{\text {def }_{M}}^{(i)}$ is faithful. The next step is showing that $\mathfrak{L} \models \psi_{\text {act }}^{(i)}$. Since $\mathfrak{L}$ is built by considering $\mathfrak{I}_{0}, \ldots, \mathfrak{I}_{n}$ that are altered by action $\mu_{0}, \ldots, \mu_{n}$, we claim that the changes represented by $\psi_{\text {act }}^{(i)}$ are satisfied. Finally, we use a similar idea to show that $\mathfrak{L} \models \psi_{\text {min }}^{(i)}$. Due to the semantics, an individual would not change in the membership of a concept name without any reason. We use the fact to show that the model built does not violate such constraint that are expressed by $\psi_{\min }^{(i)}$.

2. We unfold the model $\mathfrak{L}$ to a sequence of models $\mathfrak{I}_{0}, \ldots, \mathfrak{I}_{n}$ in a careful way. Then, we show by induction that each $C^{\mathfrak{I}_{i}}=\left(T_{C}^{(i)}\right)^{\mathfrak{L}}$ and consequently for GCIs. Next, we show for any timestamp $i$, we have that $\mathfrak{I}_{i}=\mathfrak{I}_{i-1}^{\mu_{i}}$. The base case $i=0$ is trivial. Then, we show that for each concept name (role name), the membership coincides with previous membership that altered by the effects due to the fact we restrict them using by enforcing the change in $\psi_{\text {act }}^{(i)}$ and do not let anything change without any reason with $\psi_{\min }^{(i)}$.

Lemma 1 shows the correspondence between the models of the reduction $\mathrm{m}-\mathrm{KB} \psi_{\text {red }}$ and the sequence of nested interpretations we obtain by executing $\sigma$ in a model of the initial m-KB. 
This allows one to use the following reduction of the projection problem to a (in)consistency problem in $\mathcal{A L C O} \llbracket \mathcal{A L C O} \rrbracket$.

Lemma 2. Let $\psi, \psi^{\prime}$ be $m$-KBs, and $\sigma$ be a sequence of actions. It holds that $\psi^{\prime}$ is a consequence of executing $\sigma$ in $\psi$ iff $\psi_{\text {red }} \wedge \neg\left(\psi^{\prime(n)}\right)$ is inconsistent.

Proof. $\Rightarrow$ We use a proof by contradiction. Assume that $\psi_{\text {red }} \wedge \neg\left(\psi^{\prime(n)}\right)$ is consistent. Then, there is a model $\mathfrak{L}$ such that $\mathfrak{L} \models \psi_{\text {red }}$ and $\mathfrak{L} \models \neg\left(\psi^{\prime(n)}\right)$. However, since all $\mathfrak{I}_{n} \models \psi^{\prime}$ then we have $\mathfrak{L} \models \psi^{\prime(n)}$ from Lemma 1 , hence a contradiction.

$\Leftarrow$ We use a proof by contradiction. Assume there exists an interpretation $\mathfrak{I}_{0} \models \psi$ such that $\mathfrak{I}_{0}^{\sigma}=\mathfrak{I}_{n} \not \models \psi^{\prime}$, and consequently $\mathfrak{I}_{n} \models \neg \psi^{\prime}$. By Lemma 1 , we have that $\mathfrak{L} \models \psi_{\text {red }}$ and $\mathfrak{L}=\psi^{\prime(n)}$, hence a contradiction.

This gives us a complexity result for the projection problem.

Theorem 1. The projection problem in $\mathcal{A L C O} \llbracket \mathcal{A L C O} \rrbracket$ is ExpTime-complete.

Proof. $\psi_{\text {red }}$ is polynomial in the size of the input, and obviously $\neg\left(\psi^{\prime(n)}\right)$ as well. Since the consistency problem in $\mathcal{A L C O} \llbracket \mathcal{A L C O} \rrbracket$ is ExPTIME-complete [8], we can use the following procedure: build $\psi_{\text {red }} \wedge \neg\left(\psi^{\prime(n)}\right)$ as defined, and check using an $\mathcal{A} \mathcal{L C O} \llbracket \mathcal{A L C O} \rrbracket$ consistency checker that runs in exponential time. Hence, we get an ExpTime procedure.

For hardness, we can reduce the inconsistency problem in $\mathcal{A L C O} \llbracket \mathcal{A L C O} \rrbracket$ to the projection problem. It is easy to see that an m-KB $\psi$ is inconsistent iff $\{a\} \sqsubseteq \perp$ is a consequence of executing \langle\rangle in $\psi$.

\section{Conclusion}

We have introduced an action formalism for reasoning about context and object level change in the ConDL $\mathcal{A} \mathcal{L C O} \llbracket \mathcal{A L C O} \rrbracket$. The formalism is well-behaved in the sense that the projection problem has the same complexity as standard reasoning in $\mathcal{A L C O}$.

From a practical point of view, choosing $\mathcal{A L C O} \llbracket \mathcal{A L C O} \rrbracket$ has the advantage that an efficient reasoning tool for checking consistency already exists [7]. The reasoner even supports the more expressive combination $\mathcal{S H O I} \llbracket \llbracket \mathcal{S H O I} \rrbracket$.

For future work, we plan to investigate whether our action formalism offers sufficient expressiveness for capturing also the dynamic features of the role-based modelling language in [11].

Furthermore, we would like to study the complexity of reasoning in several extensions of the action formalism. This for example includes operators for non-determinism in the action dimension and temporal specifications for possibly infinite action sequences.

\section{References}

[1] Shqiponja Ahmetaj, Diego Calvanese, Magdalena Ortiz, and Mantas Simkus. Managing change in graph-structured data using description logics. ACM Trans. Comput. Log., 18(4):27:1-27:35, 2017.

[2] Franz Baader, Diego Calvanese, Deborah L. McGuinness, Daniele Nardi, and Peter F. PatelSchneider, editors. The Description Logic Handbook: Theory, Implementation, and Applications. Cambridge University Press, 2003.

[3] Franz Baader, Ian Horrocks, Carsten Lutz, and Ulrike Sattler. An Introduction to Description Logic. Cambridge University Press, 2017. 
[4] Franz Baader, Marcel Lippmann, and Hongkai Liu. Using causal relationships to deal with the ramification problem in action formalisms based on description logics. In Christian G. Fermüller and Andrei Voronkov, editors, Logic for Programming, Artificial Intelligence, and Reasoning - 17th International Conference, LPAR-17, Yogyakarta, Indonesia, October 10-15, 2010. Proceedings, volume 6397 of Lecture Notes in Computer Science, pages 82-96. Springer, 2010.

[5] Franz Baader, Carsten Lutz, Maja Milicic, Ulrike Sattler, and Frank Wolter. Integrating description logics and action formalisms: First results. In $A A A I$, pages $572-577$. AAAI Press / The MIT Press, 2005.

[6] Charles W. Bachman and Manilal Daya. The role concept in data models. In Proceedings of the Third International Conference on Very Large Data Bases, October 6-8, 1977, Tokyo, Japan., pages 464-476. IEEE Computer Society, 1977.

[7] Stephan Böhme and Thomas Kühn. Reasoning on context-dependent domain models. In Zhe Wang, Anni-Yasmin Turhan, Kewen Wang, and Xiaowang Zhang, editors, Semantic Technology - 7th Joint International Conference, JIST 2017, Gold Coast, QLD, Australia, November 10-12, 2017, Proceedings, volume 10675 of Lecture Notes in Computer Science, pages 69-85. Springer, 2017.

[8] Stephan Böhme and Marcel Lippmann. Decidable description logics of context with rigid roles. In Carsten Lutz and Silvio Ranise, editors, Frontiers of Combining Systems - 10th International Symposium, FroCoS 2015, Wroclaw, Poland, September 21-24, 2015. Proceedings, volume 9322 of Lecture Notes in Computer Science, pages 17-32. Springer, 2015.

[9] Andreas Herzig and Ivan José Varzinczak. Metatheory of actions: Beyond consistency. Artif. Intell., 171(16-17):951-984, 2007.

[10] Szymon Klarman and Víctor Gutiérrez-Basulto. Description logics of context. J. Log. Comput., 26(3):817-854, 2016.

[11] Thomas Kühn, Stephan Böhme, Sebastian Götz, and Uwe Aßmann. A combined formal model for relational context-dependent roles. In Richard F. Paige, Davide Di Ruscio, and Markus Völter, editors, Proceedings of the 2015 ACM SIGPLAN International Conference on Software Language Engineering, SLE 2015, Pittsburgh, PA, USA, October 25-27, 2015, pages 113-124. ACM, 2015.

[12] Lars Schütze and Jerónimo Castrillón. Analyzing state-of-the-art role-based programming languages. In Jennifer B. Sartor, Theo D'Hondt, and Wolfgang De Meuter, editors, Companion to the first International Conference on the Art, Science and Engineering of Programming, Programming 2017, Brussels, Belgium, April 3-6, 2017, pages 9:1-9:6. ACM, 2017.

[13] Luciano Serafini and Martin Homola. Contextualized knowledge repositories for the semantic web. J. Web Semant., 12:64-87, 2012.

[14] Benjamin Zarrieß. Complexity of projection with stochastic actions in a probabilistic description logic. In Michael Thielscher, Francesca Toni, and Frank Wolter, editors, Principles of Knowledge Representation and Reasoning: Proceedings of the Sixteenth International Conference, KR 2018, Tempe, Arizona, 30 October - 2 November 2018., pages 514-523. AAAI Press, 2018. 